\title{
Eulerian simulations of oscillating airfoils in power extraction regime
}

\author{
G. Dumas \& T. Kinsey \\ Laval University, Quebec, Canada
}

\begin{abstract}
A wing that is both heaving and pitching simultaneously may extract energy from an oncoming flow, thus acting as a turbine. The theoretical performance of such a concept is investigated here through unsteady, two-dimensional laminar flow simulations using the finite volume, commercial CFD code FLUENT ${ }^{\mathrm{TM}}$. Computations are performed in the heaving reference frame of the airfoil, thus leaving only the pitching motion of the airfoil to be dealt with through a rigid-body mesh rotation and a circular, non-conformal sliding interface. This approach offers the benefit of second order time accurate simulations. For a NACA 0015 airfoil at a Reynolds number of $R e=1100$, a heaving amplitude of one chord $\left(H_{0}=c\right)$, and a pitching axis at the third chord $\left(x_{p}=c / 3\right)$, we present a mapping of power extraction efficiency in the frequency and pitching amplitude domain: $0<f c / U_{\infty}<0.25$ and $0<\theta_{0}<90^{\circ}$. Remarkably, efficiency as high as $34 \%$ is observed as well as a large parametric region above $\theta_{0}>55^{\circ}$ of better than $20 \%$ results. Impact of varying some of the fixed parameters is also addressed.

Keywords: oscillating wing, pitching and heaving airfoil, unsteady aerodynamics, power extraction, turbine, wind energy, flow simulation, finite volume method, accelerated reference frame.
\end{abstract}

\section{Introduction}

Following the work of McKinney and DeLaurier [1], it has been proposed in recent years to use systems of oscillating wings, heaving and pitching with large amplitudes, to develop alternative turbine designs for applications in air (wind turbine) and in water (tidal energy system). Our ongoing investigation [2] aims to establish the actual potential of the concept.

In this paper, we restrict ourselves to the canonical case of low-Reynolds number, 2-D incompressible laminar flows for which modern CFD tools can yield reli- 
able predictions at an affordable cost. This latter consideration is particularly critical here considering the great number of physical parameters involved, and thus the great number of cases that need to be computed. In addition, one should keep in mind that for a given set of parameters, the power extraction efficiency can only be evaluated once the simulation has reached its time-periodic response after several cycles of oscillation, requiring typically well above 10000 timesteps.

\section{Problem description}

We consider a symmetric airfoil undergoing a combined motion of pitching $\theta(t)$ and heaving $h(t)$ such as shown in figure 1. Restricting to a pitching axis located on the chord line at position $x_{p}$ from the leading edge, one express the airfoil motion as:

$$
\begin{aligned}
\theta(t)=\theta_{0} \sin (\gamma t) & \longrightarrow \quad \Omega(t)=\theta_{0} \gamma \cos (\gamma t) \\
h(t)=H_{0} \sin (\gamma t+\phi) & \longrightarrow \quad V_{y}(t)=H_{0} \gamma \cos (\gamma t+\phi)
\end{aligned}
$$

where $H_{0}$ and $\theta_{0}$ are respectively the heaving and pitching amplitudes, $V_{y}$ is the heaving velocity, $\Omega$ the pitching velocity, $\gamma$ the angular frequency $(=2 \pi f)$, and $\phi$ is the phase shift between the two motions which is kept fixed in this study $\left(\phi=90^{\circ}\right)$.

The effective angle of attack $\alpha$ and effective upstream velocity experienced by the airfoil during its cyclic motion are obviously functions of time. Their maximum values in the cycle are expected to have major impact on the peak forces generated, and on the probability of dynamic stall occurrence. In particular, one has $\alpha(t)=$ $\theta(t)+\arctan \left(V_{y}(t) / U_{\infty}\right)$, for which we approximate the maximum value (exact approximation in most cases) by its quarter-period value: $\alpha_{\max } \approx \alpha_{T / 4}=\theta_{0}-$ $\arctan \left(\gamma H_{0} / U_{\infty}\right)$.

One must realize that an oscillating symmetric airfoil can operate in two different regimes, namely propulsion and power extraction, depending on the value of the "feathering parameter", i.e.,

$$
\chi=\frac{\theta_{0}}{\arctan \left(H_{0} \gamma / U_{\infty}\right)} .
$$

Based on a simple quasi-steady argument [2], which leads to necessary but not precisely sufficient conditions (in a mean sense over the cycle), one can show that $\chi<1 \Rightarrow$ propulsion; $\chi>1 \Rightarrow$ power extraction. An example of the latter case is shown in the schematic representation of figure 2 which presents a time sequence viewed in a reference frame moving with the farfield flow, so that the effective angle of attack $\alpha(t)$ is made visible from the apparent trajectory of the airfoil. In that figure, $\mathbf{R}$ is first constructed from typical lift and drag forces (right-hand side), and then decomposed into $X$ and $Y$ components (left-hand side). One easily infers on figure 2 that the resultant aerodynamic force $\mathbf{R}$ would have a vertical component $Y$ that is in the same direction as the vertical displacement of the airfoil. The flow would thus make a positive work on the airfoil, and therefore, 


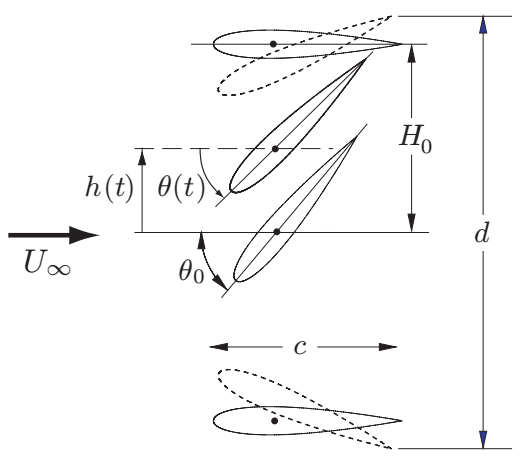

Figure 1: Imposed motion.

power would be extracted as long as no negative work is involved with respect to the horizontal component $X$. This is precisely the case of interest here since our airfoil is not moving horizontally, but only pitching and heaving into a uniform flow from left to right of speed $U_{\infty}$.

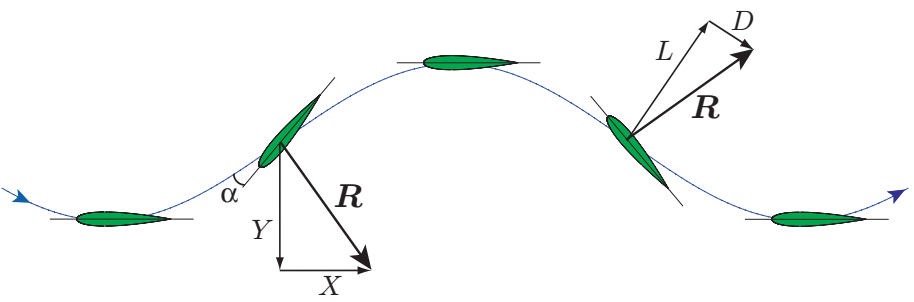

Figure 2: Power extraction regime $(\chi>1)$ of an oscillating airfoil.

\section{Numerics}

High resolution, two-dimensional unsteady computations have been performed in this study at Reynolds numbers from 500 to 2400 with the finite volume code FLUENT 6.1 [4] which allows for the use of moving meshes. Initially solving the Navier-Stokes equations in a fixed, inertial frame of reference, a proper meshing strategy taking advantage of the dynamic mesh and remeshing capabilities of the code was developed [2]. Both heaving and pitching motion of the airfoil were then taken into account through mesh motion. However, this approach in FLUENT required the use of first order time integration which therefore imposed the use of very small timestep sizes in order to control the inherent numerical diffusion.

To circumvent this constraint, a new meshing strategy has been developed in this work. In the present approach, the problem is set in a heaving reference frame (vertical translation) attached to the airfoil. This implies the use of time varying boundary conditions on exterior boundaries and the addition of the reference frame 
acceleration as a new source term in the Navier-Stokes equation. The pitching portion of the airfoil motion on the other hand is left as such, i.e., the airfoil is actually pitching in the heaving reference frame, and this body motion is taken into account by the use of a moving mesh involving a circular, non-conformal sliding interface. As can be seen on figure 3, this interface is located at five chords around the airfoil, and the grid inside is pitching in rigid body with the airfoil. The grid outside the interface is not moving. This strategy offers the significant advantage of allowing for the use of second order time integration scheme rather than only first order.

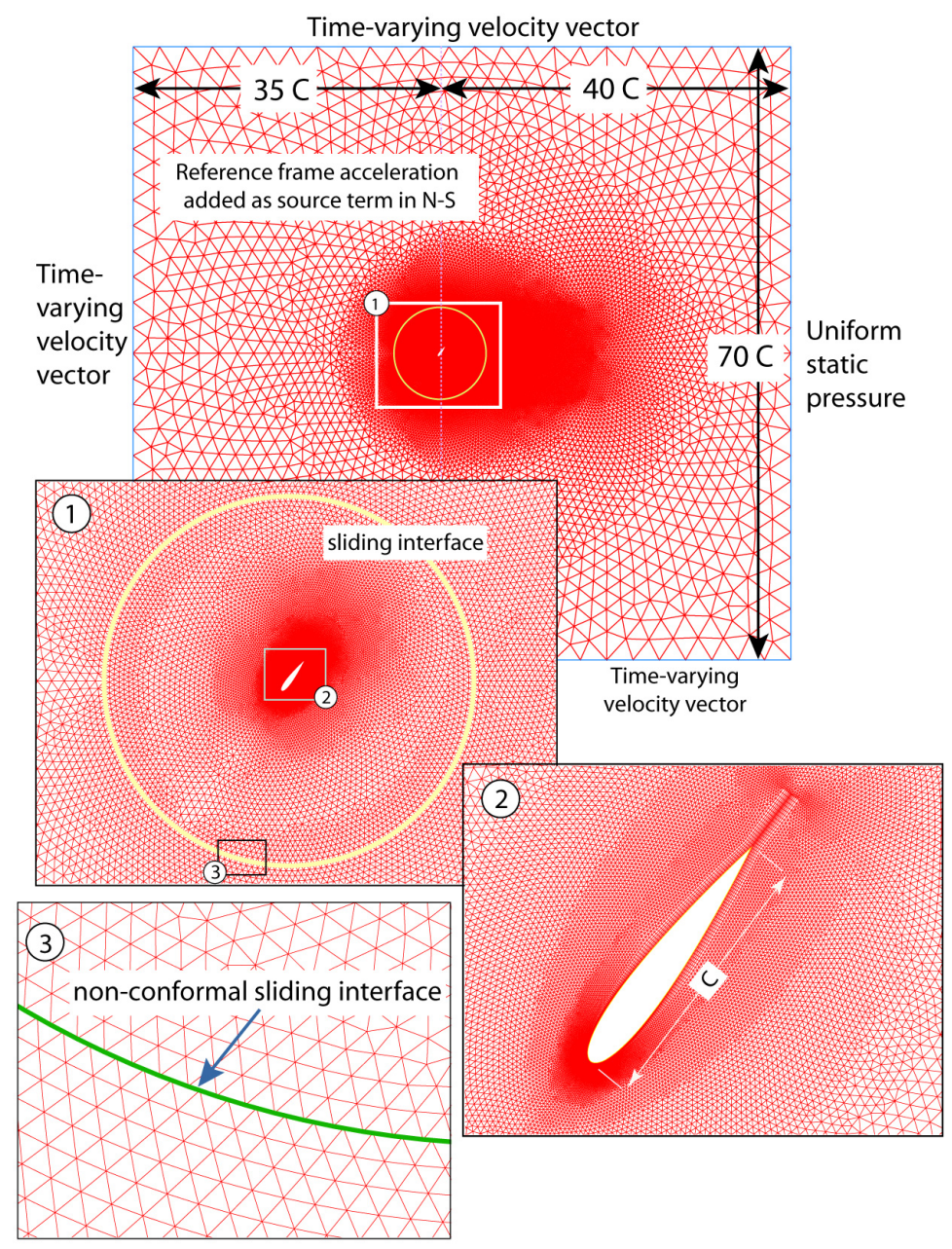

Figure 3: Grid details with two zoom levels showing its circular, non-conformal sliding interface (typical grid size: 72000 cells). 
In order to reach the long-term, periodic flow response after the impulsive start in each of our simulations, a few cycles are first computed with a relaxed timestep size. The precise number of cycles computed at that stage depends on the period of oscillation $T=1 / f$. This transitory period should provide at least 20 convective time units, i.e., time enough for the early wake to get sufficiently far away from the body. From that point on, we compute more cycles using a finer time resolution given by

$$
\Delta t=\min \left\{\frac{T}{2000} ; \frac{c /\|V\|}{100}\right\}
$$

where $\|V\|$ is a velocity norm representing the maximum instantaneous convective flux velocity in the domain which takes into account $U_{\infty}$, the heaving boundary condition and the pitching mesh velocity. Normalized flow diagnostics are thereafter monitored so as to assert the periodicity of the final cycles. A criterion of less than $0.5 \%$ variation in mean statistics between final cycles is typically used. Flow quantities and aerodynamic forces of the last cycle are then used to compute mean values as well as efficiency. Typical run time for a whole simulation is about 100 hours on a single $\mathrm{P} 4 / 3.2 \mathrm{GHz}$ processor.

A great deal of attention and rigor has been paid in this numerical investigation to assure good prediction accuracy throughout the targeted parametric space. Several auto-validation tests were carried out until force predictions independency was satisfactorily achieved with respect to all modelling aspects: mesh refinement and isotropy near the body, grid relaxation away from the airfoil, timestep size, domain size, periodicity criterion, sliding interface position, as well as implementation of user-defined functions (UDF) for unsteady boundary conditions and unsteady momentum source terms. In addition, numerous comparisons with several other studies were realized, in particular with the works of Blackburn and Henderson [5], Ohmi et al. [6], Jones et al. [3], and Pedro et al. [7]. Very good agreement was in general achieved, or, if need be, discrepancies successfully explained. This will be reported separately elsewhere (but in full details in: T. Kinsey, 2006, M.Sc. thesis, Laval University).

\section{Results}

The instantaneous power extracted from the flow (per unit depth) when $\chi>1$ comes from the sum of a heaving contribution $P_{y}(t)=Y(t) V_{y}(t)$ and a pitching contribution $P_{\theta}(t)=M(t) \Omega(t)$, where $M$ is the resulting torque about the pitching center $x_{p}$. The mean power extracted over one cycle can thus be computed in non-dimensional form $\left(C_{P} \equiv \frac{P}{\frac{1}{2} \rho U_{\infty}^{3} c}\right)$ as:

$$
\bar{C}_{P}=\bar{C}_{P_{y}}+\bar{C}_{P_{\theta}}=\int_{0}^{1}\left\{C_{Y}(t) \frac{V_{y}(t)}{U_{\infty}}+C_{M}(t) \frac{\Omega(t) c}{U_{\infty}}\right\} d(t / T) .
$$

We further define the power extraction efficiency $\eta$ as the ratio of the mean total power extracted $\bar{P}$ to the total power available $P_{a}$ in the oncoming flow passing 
through the swept area (the "flow window"):

$$
\eta \equiv \frac{\bar{P}}{P_{a}}=\frac{\bar{P}_{y}+\bar{P}_{\theta}}{\frac{1}{2} \rho U_{\infty}^{3} d}=\bar{C}_{P} \frac{c}{d}
$$

where $d$ is the overall, vertical extent of the airfoil motion (see figure 1).

\subsection{Efficiency — basic case}

A partial mapping of the predicted efficiencies (over 42 simulations) in the parametric space $\left(f^{*}, \theta_{0}\right)$ is provided in figure 4 for our basic case: NACA 0015 airfoil, $R e=U_{\infty} c / \nu=1100, H_{0} / c=1$ and $x_{p} / c=1 / 3$. Note that the dimensionless frequency is defined here as $f^{*} \equiv f c / U_{\infty}$.

First, we find that the highest efficiency achieved, $\eta_{\max } \approx 34 \%$, is obtained for high pitching amplitudes, $\theta_{0} \approx 70-80^{\circ}$, and at non-dimensional frequencies in the range $f^{*} \approx 0.12-0.18$. As a reference, let us recall here the theoretical limit of Betz (from actuator disk theory) at $59 \%$ which should apply to the present

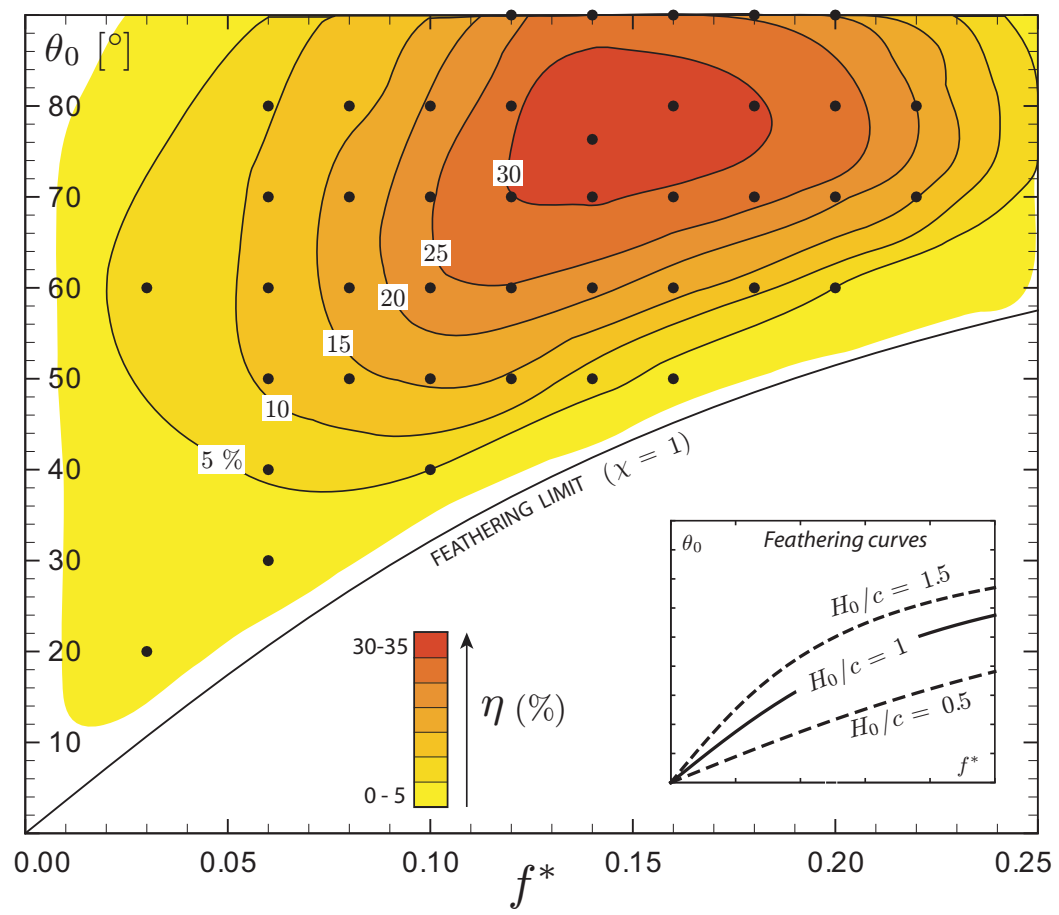

Figure 4: Preliminary mapping of efficiency $\eta$ in the parametric space $\left(f^{*}, \theta_{0}\right)$ for a NACA 0015 at $R e=1100, H_{0} / c=1$ and $x_{p} / c=1 / 3$. Simulated cases are shown with black circles. Note that the iso-efficiency contours have been sketched approximately. 
oscillating wing problem since a cycle-averaged, stationary flow concept may be defined. In any case, efficiencies higher than $30 \%$ for a single oscillating airfoil appear quite encouraging from a practical point of view for the present turbine concept.

We further note that the best efficiency cases in figure 4 correspond to operating conditions of roughly $V_{y_{\max }} / U_{\infty}=2 \pi f^{*} H_{0} / c \approx 1$ (maximum heaving velocity comparable to free stream velocity). At the same time, these cases are found to involve effective angles of attack (see Section 2) reaching as high as $35^{\circ}$ during their cycles. With such large values of angle of attack, it is no surprise to observe some dynamic stall vortex shedding taking place during the motion. Indeed, leading edge vortex shedding (LEVS) is seen to occur during the cycles of most of the efficient cases (see figure 5). In fact, one finds that well-timed LEVS, occurring each half period just prior to $t \approx 0$ and $t \approx T / 2$, is a very important mechanism to maximize the power extraction efficiency as can be illustrated with the help of figure 5 .

The case shown in figure 5a corresponds to a typically smooth aerodynamic flow with a moderate efficiency of $\eta=11 \%$ while the case of figure $5 \mathrm{~b}$, which is at a slightly lower frequency but higher pitching amplitude, exhibits dynamic stall vortex shedding and reaches a mean efficiency of nearly $34 \%$. The straight horizontal line corresponding to the theoretically available power $C_{P_{a}}$ has been added to the figures as a reference level.

The main feature revealed by figure 5 is that case (a), without LEVS, shows a poor synchronization between $V_{y}$ and $C_{Y}$ (i.e., exhibiting opposite signs at times) causing the total power curve to go negative in some parts. On the other hand, case (b) exhibiting dynamic stall, shows good timing in the sign switch of $V_{y}$ and $C_{Y}$, resulting in positive values of total extracted power over almost all of the cycle. It is clearly the shed vortices and their suction effect on the airfoil that are responsible for maintaining a negative $Y$ force much closer to the mid-cycle time at $t / T=0.5$. In this particular case, one notes further that the shedding at each half-cycle has also a favourable impact on the pitching contribution $P_{\theta}$ which can play a positive role momentarily in the cycle, despite its small overall mean contribution.

Indeed, one finds in this study that for most cases of interest, the heaving contribution $P_{y}$ significantly dominates the pitching contribution $P_{\theta}$. One may thus write $P(t) \approx Y(t) V_{y}(t)$. Consequently, one concludes that there are three major aspects affecting the level of power extracted:

- synchronization between the vertical force $Y(t)$ and the heaving velocity $V_{y}(t) \longrightarrow$ both have to be of the same sign most of the cycle to avoid negative power occurrences;

- the magnitude of the heaving velocity $V_{y}(t) \longrightarrow$ being proportional to $H_{0}$ and $f$, see Eq.(2), higher frequencies and/or higher heaving amplitude appear favourable;

- the magnitude of the vertical force $Y(t) \longrightarrow$ complex dependency on both maximum effective angle of attack and maximum effective velocity.

It is clear that increasing the effective velocity by increasing $V_{y}$ (through $H_{0} / c$ and/or $f^{*}$ ) leads to increased effective dynamic pressure, and thus increased aero- 
(a)

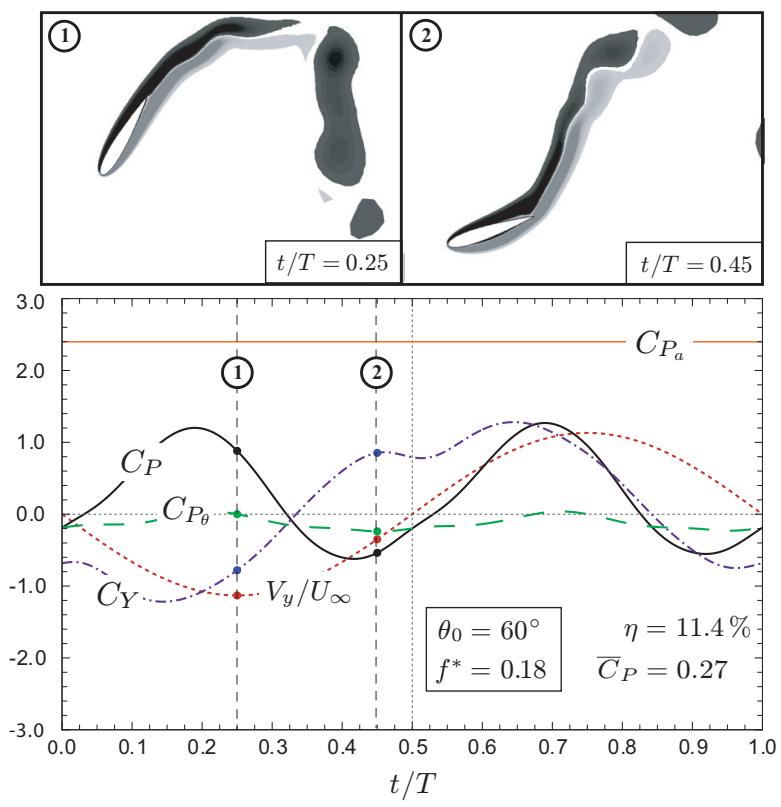

(b)

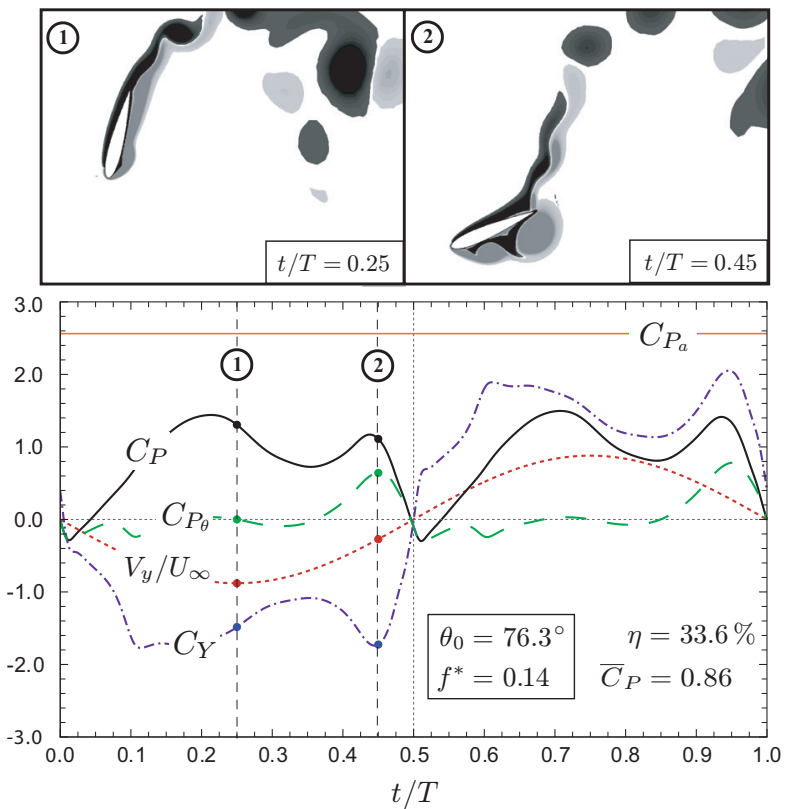

Figure 5: Contours of vorticity at two instants in the cycle (top), and time evolution (bottom) of total power coefficient $C_{P}-$ as well as $V_{y} / U_{\infty}, C_{Y}$, and $C_{P_{\theta}}$ - for two power extraction cases of figure 4. (a) A moderate efficiency case: No leading edge vortex shedding (LEVS); poor synchronization of $Y$ vs $V_{y}$. (b) A typical high efficiency case: Occurrence of LEVS; optimal synchronization. 
dynamic forces. However, the vertical force component $Y$ may at the same time start decreasing due to decreasing effective angle of attack and reduced vertical projection. Indeed, increasing either the frequency $f$ or the heaving amplitude $H_{0}$ (everything else being the same) inevitably moves us towards the feathering limit, which ultimately has a detrimental effect past a certain level. As suggested in figure 4 , there has to be an optimum in those parameters.

\subsection{Parametric study — preliminary}

An extensive parametric investigation is currently underway in order to qualify, and quantify as much as possible, the effects of all the parameters involved in the characterization of an oscillating wing in power extraction. Several of the numerous parameters that had been set fixed are now varied: heaving amplitude $H_{0}$, pitching axis location $x_{p}$, airfoil geometry, phase angle $\phi$, heaving and pitching functions, and Reynolds number. Preliminary results briefly discussed below concern the effects of airfoil thickness, heaving amplitude and $R e$ number.

What we find with respect to airfoil thickness (NACA 0002 up to NACA 0020) is that global efficiency is little sensitive to the details of the geometry. The aerodynamics at play here is very much inertial, and governed by the forced, large amplitude oscillation. Although dynamic stall, thus boundary layer separation, is seen to play an important role in some cases, the precise location of flow separation along the airfoil is apparently not so critical. Indeed, for airfoil thickness varying from $2 \%$ to $20 \%$ chord, we obtain for the case of figure 5 (a), $11.0 \leq \eta \leq 11.6 \%$, while for the case of figure 5(b), efficiency varies slightly more in the range $32.0 \leq$ $\eta \leq 33.6 \%$. Again, from a practical point of view, these observations are rather favourable and encouraging.

Table 1: Effect of a larger heaving amplitude.

\begin{tabular}{|c|c|c|c|c|}
\hline Case & \multicolumn{2}{|c|}{$H_{0} / c=1.0$} & \multicolumn{2}{c|}{$H_{0} / c=1.5$} \\
\hline$\left(f^{*}, \theta_{0}\right)$ & $\bar{C}_{P}$ & $\eta(\%)$ & $\bar{C}_{P}$ & $\eta(\%)$ \\
\hline$\left(0.18,60.0^{\circ}\right)$ & 0.27 & 11.4 & -0.69 & - \\
\hline$\left(0.14,76.3^{\circ}\right)$ & 0.86 & 33.6 & 0.98 & 28.5 \\
\hline
\end{tabular}

The impact of varying the heaving amplitude is much more complex and significant since it affects directly the oscillating velocities (at a given frequency), thus inertia, and the size of the flow window swept by the airfoil. Referring again to the two basic cases considered in figure 5, we find that although efficiency tends to decrease with an increase in amplitude (table 1), it may not be so for the power coefficient. This point would have to be kept in mind when applying the concept in practice. Table 1 also provides an example of a $\chi>1$ case, thus above its feathering limit (but only slightly), that does not, in the mean, extract power from the flow 
$\left(\bar{C}_{P}<0\right)$. As mentioned previously, actual operating conditions have to remain away from the feathering limit.

Finally, the effect of viscosity has been addressed by simulating again our two basic cases of figure 5 for a lower (500) and a larger (2400) Re number. We find that efficiency tends to increase slightly with $R e$, from 9.8 to $11.9 \%$ for case (a), and from 32.7 to $36 \%$ for case (b). It is expected that at even higher $R e$ numbers, turbulence might have a more significant impact on the power extraction efficiency. To address this, we plan to conduct URANS simulations in the near future.

\section{Conclusion}

The power extraction potential of an oscillating airfoil has been investigated in this study. For low-Re number and 2-D flow conditions, it has been shown that efficiencies as high as $34 \%$ can be obtained for reduced frequency $f^{*} \approx 0.15$ and under high pitching amplitude $\theta_{0} \approx 75^{\circ}$. For the parameters considered, efficiencies above $20 \%$ require a minimum pitching amplitude of about $55^{\circ}$. Dynamic stall vortices have also been observed to play a key role in achieving optimal efficiency. It has also been confirmed that the physics of such oscillating airfoils is dominated by the imposed motion of large amplitude, and very little sensitive (in terms of efficiency) to the airfoil thickness and the level of viscous diffusion.

\section{Acknowledgements}

Financial support from NSERC Canada is gratefully acknowledged.

\section{References}

[1] McKinney W. \& DeLaurier J., The Wingmill: An Oscillating-Wing Windmill, J. of Energy, Vol. 5, No.2, pp. 109-115, 1981.

[2] Dumas G. \& Kinsey T., Unsteady Forces on Flapping Airfoils, Paper CASI257, 11th Aerod. Symp., Canadian Aeronautics and Space Institute, April 2627, 2005, Toronto, Canada.

[3] Jones, K.D., Lindsey, K. and Platzer, M.F., An Investigation of the FluidStructure Interaction in an Oscillating-Wing Micro-Hydropower Generator, Fluid Structure Interaction II, Chakrabarti, Brebbia, Almorza and GonzalezPalma Eds., WIT Press, pp. 73-82, 2003.

[4] Fluent 6.1 User's Guide, Fluent Inc. 2003, http://www.fluent.com

[5] Blackburn H. M. \& Henderson R. D., A study of two-dimensional flow past an oscillating cylinder, J. of Fluid Mech. 385, pp. 255-286, 1999.

[6] Ohmi K., Coutanceau M., Daube O. and Loc T. P., Further experiments on oscillating airfoils, J. of Fluid Mech. 225, pp. 607-630, 1991.

[7] Pedro G., Suleman A. and Djilali N., A numerical study of the propulsive efficiency of a flapping hydrofoil, Int. J. Numer. Meth. Fluids, Vol. 42, 493-526, 2003. 